\title{
XMM-Newton and Chandra Observations of Neutron Stars and Cataclysmic Variables in the Globular Cluster NGC 2808
}

\author{
M. Servillat ${ }^{1}$, N. A. Webb $^{1}$, D. Barret ${ }^{1}$, R. Cornelisse $^{2}$, A. Dieball ${ }^{3}$, \\ C. Knigge ${ }^{3}$, K. S. Long ${ }^{4}$, M. M. Shara ${ }^{5}$ and D. R. Zurek \\ ${ }^{1}$ CESR, CNRS, Université Paul Sabatier, Toulouse, France \\ email: mathieu.servillat@cesr.fr \\ ${ }^{2}$ Instituto de Astrofisica de Canarias, Santa Cruz de Tenerife, Spain \\ ${ }^{3}$ School of Physics and Astronomy, University of Southampton, UK \\ ${ }^{4}$ Space Telescope Science Institute, Baltimore, USA \\ ${ }^{5}$ Department of Astrophysics, American Museum of Natural History, New York, USA
}

\begin{abstract}
.
We report on XMM-Newton and Chandra observations of the globular cluster NGC 2808. We detect one quiescent low mass X-ray binary of the $3 \pm 1$ expected, if these systems are formed through encounters, and we show evidence for the presence of $20 \pm 10$ bright cataclysmic variables in the core with a luminosity above $4 \times 10^{31} \mathrm{erg} \mathrm{s}^{-1}$. We also review the specific nature of cataclysmic variables in globular clusters with reference to recent VLT/FORS1 observations of a cataclysmic variable in M 22 .
\end{abstract}

Keywords. globular clusters: individual (NGC 2808, M 22), stars: neutron, cataclysmic variables, binaries: close

Globular clusters (GCs) are thought to harbour a large number of interacting binaries which are believed to delay their core collapse. With XMM-Newton and Chandra, it is possible to detect faint X-ray sources such as active binaries (ABs) and interacting binaries and their products, e.g. neutron star low mass X-ray binaries often in quiescence (qLMXBs), cataclysmic variables (CVs) and millisecond pulsars (MSPs). We discuss here only the core X-ray sources of NGC 2808 (for a discussion of XMM-Newton X-ray sources outside NGC2808's core, see Servillat, Webb \& Barret 2008).

Based on the colour-flux diagram of all the detected sources (Fig. 1), we identify some of the core sources:

1 qLMXB. $3 \pm 1$ qLMXBs are expected if they are formed through encounters (Gendre et al. 2003). C2 is the only qLMXB candidate discovered with XMM-Newton (Servillat, Webb \& Barret 2008). The spectrum of C2, after subtracting the contribution of source $\mathrm{C} 1$, is well fitted with a hydrogen atmosphere and has parameters typical for neutron stars. Chandra observations confirm the XMM-Newton identification.

$\sim 9$ bright CVs. From observations of 23 GCs (Pooley \& Hut 2006), we expect to detect $20 \pm 10 \mathrm{CVs}$ with luminosities above $4 \times 10^{31} \mathrm{erg} \mathrm{s}^{-1}$ in NGC 2808, which is consistent with the global luminosity of the core (without C2). C1 is resolved into 4 Chandra sources, all consistent with CV emission. The XMM-Newton spectrum of C1 is well fitted with a high temperature bremsstrahlung model, typical of CVs (Baskill et al. 2005). C3 has faded by almost a factor 10 in the Chandra observation. C5 is undetected with Chandra, and one Chandra source was not detected with XMM-Newton, implying a flux variation of at least a factor 10 . 


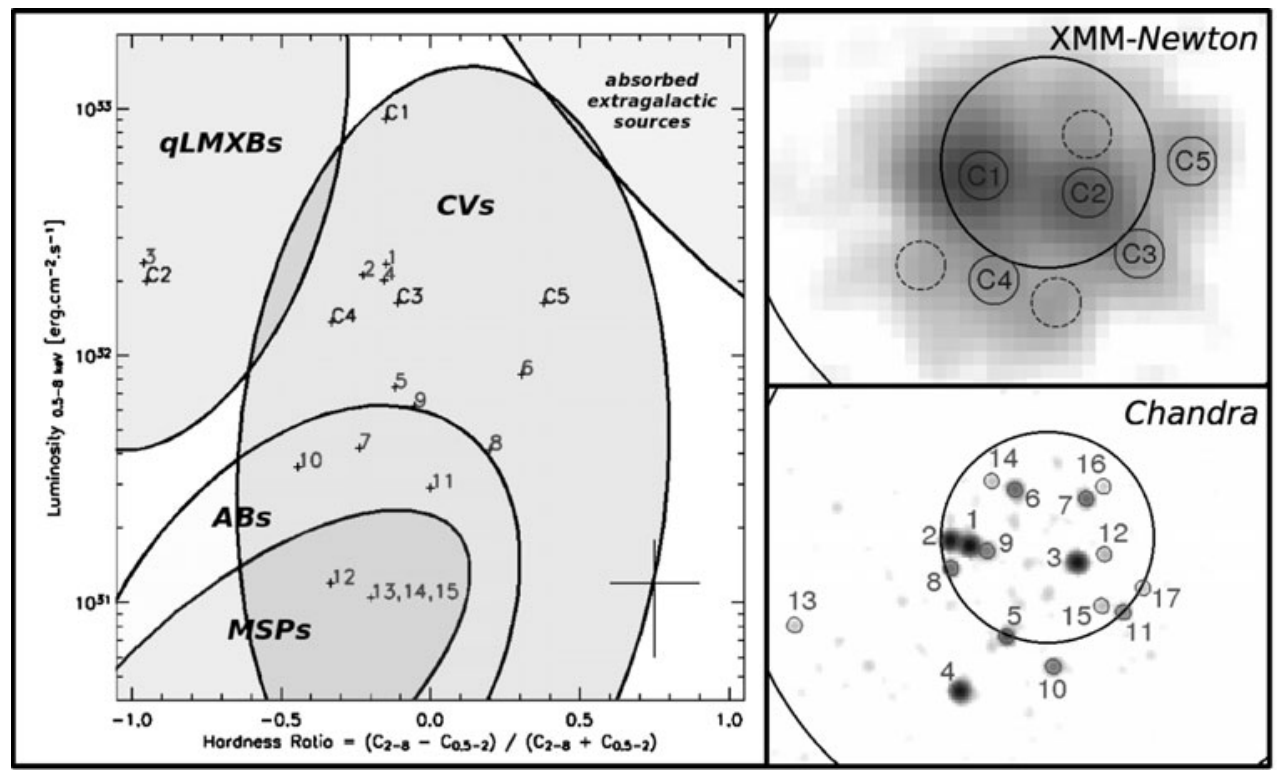

Figure 1. Left: colour-flux diagram of detected sources. It is divided into very approximate regions extrapolated from Pooley \& Hut 2006. Right: images of the core of NGC 2808 from XMM-Newton and Chandra observations, where the core and half-mass radii are plotted.

- We note that the XMM-Newton and Chandra observations are complementary and well correlated.

- We find evidence for CV variability and maybe as many as three outbursts.

- The spectrum of $\mathrm{C} 2$ is helpful for constraining the neutron star equation of state as described in Webb \& Barret (2007).

- Few outbursts have been detected in GC CVs compared to field CVs, leading to the idea that the two populations may harbour different properties. It was proposed that GC $\mathrm{CVs}$ have moderately strong magnetic fields that stabilize the accretion flow (Dobrotka et al. 2006). The mass of the white dwarf is also possibly higher in GC CVs (Ivanova et al. 2006). Another possibility is that because of crowding in GCs, many CV outbursts were not detected. From our two sets of X-ray observations of NGC 2808, we detect high flux variations for three CV candidates, possibly three outbursts. Two CVs in M22 have also been observed in outburst optically. From recent optical spectroscopy of these faint CVs with VLT/FORS1, we will determine the mass of the white dwarf and the magnetic field to determine whether these play a role in $\mathrm{GC} \mathrm{CVs}$.

- Ultra-violet data (Dieball et al. 2005) and other multi-wavelength observations will help in identifying the faintest sources (Servillat et al. in preparation).

\section{References}

Baskill, D. S., Wheatley, P. J. \& Osborne, J. P. 2005, MNRAS 357, 626

Dieball, A., Knigge, C., Zurek, D. R., Shara, M. M. \& Long, K. S. 2005, ApJ 625, 156

Dobrotka, A., Lasota, J-P. \& Menou, K. 2006, ApJ 640, 288

Gendre, B., Barret, D., \& Webb, N. 2003, A\& A, 403, L11

Ivanova, N., Heinke, C. O., Rasio, F. A., et al. 2006, MNRAS 372, 1043

Pooley, D. \& Hut, P. 2006, ApJ 646, L143

Servillat, M., Webb, N. A. \& Barret, B., 2008, A\&\&A submitted

Webb, N. A. \& Barret, D. 2007, ApJ in press, arXiv:0708.3816 